\title{
Radionuclide Therapy of HER2-Expressing Human Xenografts Using Affibody-Based Peptide Nucleic Acid-Mediated Pretargeting: In Vivo Proof of Principle
}

\author{
Kristina Westerlund*1, Mohamed Altai*2, Bogdan Mitran ${ }^{3}$, Mark Konijnenberg ${ }^{4}$, Maryam Oroujeni ${ }^{2}$, Christina Atterby ${ }^{2}$, \\ Marion de Jong ${ }^{4}$, Anna Orlova ${ }^{3}$, Johanna Mattsson ${ }^{2}$, Patrick Micke ${ }^{2}$, Amelie Eriksson Karlström ${ }^{\dagger 1}$, and \\ Vladimir Tolmachev ${ }^{\dagger 2}$ \\ ${ }^{I}$ Department of Protein Science, KTH Royal Institute of Technology, Stockholm, Sweden; ${ }^{2}$ Department of Immunology, Genetics, and \\ Pathology, Uppsala University, Uppsala, Sweden; ${ }^{3}$ Division of Molecular Imaging, Department of Medicinal Chemistry, Uppsala \\ University, Uppsala, Sweden; and ${ }^{4}$ Department of Radiology and Nuclear Medicine, Erasmus MC, Rotterdam, The Netherlands
}

\begin{abstract}
Affibody molecules are small proteins engineered using a nonantibody scaffold. Radiolabeled Affibody molecules are excellent imaging probes, but their application to radionuclide therapy has been prevented by high renal reabsorption. The aim of this study was to test the hypothesis that Affibody-based peptide nucleic acid (PNA)-mediated pretargeted therapy of human epidermal growth factor receptor 2 (HER2)-expressing cancer extends survival without accompanying renal toxicity. Methods: A HER2-targeting Affibody molecule ligated with an AGTCGTGATGTAGTC PNA hybridization

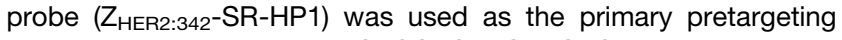
agent. A complementary AGTCGTGATGTAGTC PNA conjugated to the chelator DOTA and labeled with the radionuclide ${ }^{177} \mathrm{Lu}$ $\left({ }^{177} \mathrm{Lu}-\mathrm{HP} 2\right)$ was used as the secondary agent. The influence of different factors on pretargeting was investigated. Experimental radionuclide therapy in mice bearing SKOV-3 xenografts was performed in 6 cycles separated by $7 \mathrm{~d}$. Results: Optimal tumor targeting was achieved when $16 \mathrm{MBq} / 3.5 \mu \mathrm{g}(0.65 \mathrm{nmol})$ of ${ }^{177} \mathrm{Lu}-\mathrm{HP} 2$ was injected $16 \mathrm{~h}$ after injection of $100 \mu \mathrm{g}(7.7 \mathrm{nmol})$ of $Z_{\text {HER2:342-SR-HP1. The }}$ calculated absorbed dose to tumors was $1,075 \mathrm{mGy} / \mathrm{MBq}$, whereas the absorbed dose to kidneys was $206 \mathrm{mGy} / \mathrm{MBq}$ and the absorbed dose to blood (surrogate of bone marrow) was $4 \mathrm{mGy} / \mathrm{MBq}$. Survival of mice was significantly longer $(P<0.05)$ in the treatment group $(66 \mathrm{~d})$ than in the control groups treated with the same amount of

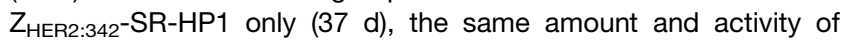
${ }^{177}$ Lu-HP2 only (32 d), or phosphate-buffered saline (37 d). Conclusion: The studied pretargeting system can deliver an absorbed dose to tumors appreciably exceeding absorbed doses to critical organs, making Affibody-based PNA-mediated pretargeted radionuclide therapy highly attractive.
\end{abstract}

Key Words: pretargeting; PNA; affibody molecules; radionuclide therapy; HER2

J Nucl Med 2018; 59:1092-1098

DOI: 10.2967/jnumed.118.208348

\footnotetext{
Received Jan. 16, 2018; revision accepted Jan. 23, 2018

For correspondence or reprints contact: Vladimir Tolmachev. Department of Immunology, Genetics, and Pathology, Uppsala University, SE-75181, Uppsala, Sweden.

E-mail: vladimir.tolmachev@igp.uu.se

${ }^{*}$ Contributed equally to this work.

${ }^{\dagger}$ Contributed equally to this work.

Published online Feb. 9, 2018.

COPYRIGHT (C 2018 by the Society of Nuclear Medicine and Molecular Imaging.
}

$\mathbf{T}$ he concept of pretargeted radionuclide therapy has evolved since late 1980s in response to the limited success of radioimmunotherapy (1). Radiolabeled monoclonal antibodies brought about an impressive improvement in survival for hematologic malignancies but failed to deliver therapeutically meaningful absorbed doses to solid tumors without unacceptably high absorbed doses to radiosensitive tissues (2). The major problems, that is, the long residence time of antibodies in blood and their inefficient penetration into tumors, are associated with the bulkiness of intact immunoglobulins.

In pretargeting, the process of molecular recognition of tumorassociated antigens is separated from delivery of radionuclide. In a first phase, a primary agent (e.g., an antibody conjugated with a recognition tag) binds to cancer cells, and nonbound primary agent is then cleared from blood. A small radiolabeled secondary agent with high affinity to the recognition tag on the primary agent is injected afterward. The small size should ensure rapid penetration into tumors and efficient renal clearance of free radioconjugate (3). Several approaches to pretargeting are under evaluation (4).

For a long time, antibodies were considered to be the only class of proteins providing specific high-affinity binding. However, nonimmunoglobulin engineered scaffold proteins (ESPs) have appeared as a strong complement to or even substitute for antibodies in treatments requiring molecular recognition (5). ESP contains a robust scaffold part, which supports variable amino acids. The presence of variable amino acids permits development of extensive combinatorial libraries enabling selection of binders with high specificity to a designated target. The scaffold part reduces entropic penalty and provides high affinities. Importantly, ESPs can be much smaller than antibodies, therefore solving issues of extravasation, homogeneous distribution inside tumors, and clearance of unbound targeting agent from blood (5). ESPs, unlike antibodies, can be produced in prokaryotes, increasing their yield appreciably and reducing production costs-a major advantage for clinical translation (6). Several ESPs, such as Affibody (Affibody AB) molecules, fibronectins, DARPins (Molecular Partners), and knottins, have demonstrated promising features as targeting probes for radionuclide molecular imaging (6). However, application of many of these ESPs for radionuclide therapy has been complicated by the high renal reabsorption of proteins after 
glomerular filtration (7-10). We hypothesized that pretargeting using a secondary hapten with low renal reabsorption might result in safe ESP-based radionuclide therapy. To test this hypothesis, we used a human epidermal growth factor receptor 2 (HER2)-targeting Affibody molecule as a model.

The scaffold of Affibody molecules is based on the 58-amino-acid domain B of protein A (11). Affibody molecules that bind with high affinity to several cancer-associated targets have been selected (12). HER2 is overexpressed in about $20 \%$ of breast and $15 \%$ of gastric cancer and is a predictive biomarker for response to treatment with the HER2-specific monoclonal antibody trastuzumab $(13,14)$. The antiHER2 Affibody molecule ABY-025 demonstrated efficient and specific imaging of HER2-expressing breast cancer metastases in clinical studies $(15,16)$. Importantly, both preclinical and clinical studies have demonstrated that Affibody molecules lack toxicity or immunogenicity. However, renal uptake of radiometal-labeled Affibody molecules was clearly higher than tumor uptake, preventing their safe application for radionuclide therapy $(7,15,16)$. Renal reabsorption of Affibody molecules is megalin-independent (17). Conventional methods of reducing renal uptake of radiopeptides, such as coinjection or preinjection of cationic amino acids or succinylated gelatin (Gelofusine; Hausmann Laboratories Ltd.), are inefficient for Affibody molecules (17).

Hybridization of complementary peptide nucleic acids (PNA) was selected as one of the approaches to Affibody-based pretargeting. PNA is a nucleic acid analog capable of Watson-Crick base pairing (18) that can easily be produced in a good-manufacturing-practicecompatible way by solid-phase peptide synthesis. PNA is based on an $N$-(2-aminoethyl)-glycine pseudopeptide chain (Fig. 1A) instead of a sugar-phosphate backbone, and because of the backbone substitution it is degraded by neither nucleases nor peptidases. PNAs are nontoxic, nonimmunogenic, and capable of hybridization with complementary PNAs in vivo (19-21). We have developed the complementary PNA hybridization probes HP1 and HP2, conjugated with the chelator DOTA for radiometal labeling, and established a methodology for site-specific sortase A catalyzed ligation of HP1 to the HER2-targeting Affibody molecule $\mathrm{Z}_{\mathrm{HER} 2: 342}$, forming the $\mathrm{Z}_{\mathrm{HER} 2: 342}$-SR-HP1 Affibody-PNA chimera (Figs. 1B and 1C) (22). $\mathrm{Z}_{\mathrm{HER} 2: 342-\mathrm{SR}-\mathrm{HP} 1 \text { binds }}$ to HER2-expressing cells specifically with an affinity of $6 \pm 2 \mathrm{pM}$, and radiolabeled HP2 binds to $\mathrm{Z}_{\mathrm{HER} 2: 342}$-SR-HP1-pretreated cells with an affinity of about $300 \mathrm{pM}(23)$. HER2-specific accumulation of ${ }^{111} \mathrm{In}-\mathrm{Z}_{\mathrm{HER} 2: 342}$-SR-HP1 and pretargeting-dependent uptake of

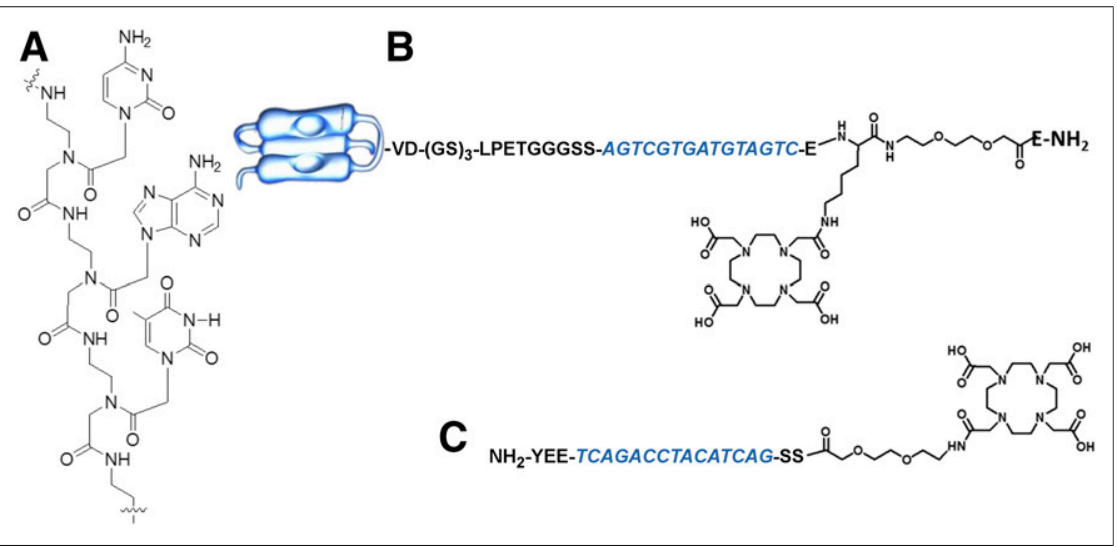

FIGURE 1. (A) Example of PNA structure. (B) HP1 hybridization probe ligated to Affibody molecule (ZHER2:342-SR-HP1) and DOTA. (C) HP2 complementary hybridization probe with DOTA. One-letter codes are applied for designation of both amino acid and nitrogenous bases. PNA part is in blue font.
${ }^{111}$ In-HP2 in HER2-expressing xenografts have also been demonstrated (23). Importantly, we found that tumor uptake of ${ }^{111} \mathrm{In}-\mathrm{HP} 2$ was twice the renal uptake after pretargeting. Although this difference was insufficient for tumor treatment without damaging the kidneys, the renal uptake was already reduced 20-fold compared with nonpretargeted radiometal-labeled Affibody molecules, suggesting that a further optimized pretargeting approach might lead to safe and effective Affibody-based radionuclide therapy. Moreover, efficient labeling of HP2 with the therapeutic radionuclide ${ }^{177} \mathrm{Lu}$ has been established (24).

The aim of this study was to evaluate factors influencing Affibody-based ${ }^{177} \mathrm{Lu}-\mathrm{HP} 2$ pretargeting and to test the hypothesis that Affibody-based pretargeted therapy can prevent renal toxicity and improve survival in mice bearing HER2-expressing xenografts.

\section{MATERIALS AND METHODS}

\section{Targeting Probes}

$\mathrm{Z}_{\mathrm{HER} 2: 342}$-SR-HP1 and HP2 were produced and purified as described earlier $(22,24)$. Briefly, the PNA hybridization probes, HP1 and HP2, were created using manual Fmoc-protected solid-phase synthesis on a Rink-Amide ChemMatrix resin (Biotage) with commercially available building blocks. HP1 was covalently and sitespecifically ligated to an anti-HER2 Affibody using an enzymatic, sortase A-mediated ligation. The Affibody construct, $\mathrm{Z}_{\mathrm{HER} 2: 342}$-SR$\mathrm{H}_{6}$, was expressed in Escherichia coli and purified using standard protocols for $\mathrm{His}_{6}$-tagged proteins before ligation. The targeting agent $\mathrm{Z}_{\mathrm{HER} 2: 342}$-SR-HP1 and the secondary agent HP2 were purified on a reversed-phase high-performance liquid chromatography column using a water/acetonitrile gradient with $0.1 \%$ trifluoroacetic acid. The final purity of $\mathrm{Z}_{\mathrm{HER} 2: 342}$-SR-HP1 and HP2 was at least $95 \%$ as judged by analytic reversed-phase high-performance liquid chromatography.

For labeling, a solution of HP2 in $1 \mathrm{M}$ ascorbic acid, pH $5.5(0.5 \mu \mathrm{g} / \mu \mathrm{L})$, was mixed with ${ }^{177} \mathrm{Lu}$-chloride (4.8 MBq/ $\mu \mathrm{g}$ of HP2). The mixture was incubated at $95^{\circ} \mathrm{C}$ for $60 \mathrm{~min}$, and radiochemical purity was measured using radio-instant-thin layer chromatography eluted with $0.2 \mathrm{M}$ citric acid, $\mathrm{pH}$ 2.0. The purity of $\mathrm{Z}_{\mathrm{HER} 2: 342^{-S R}-\mathrm{HP} 1 \text { was over }}$ $95 \%$. The radiochemical purity of ${ }^{177} \mathrm{Lu}-\mathrm{HP} 2$ was over $98 \%$, and the specific activity was $24.7 \mathrm{GBq} / \mu \mathrm{mol}$.

\section{Optimization of Pretargeting}

Animal experiments were performed in accordance with the national legislation for work with laboratory animals. Approval was granted by the Ethical Committee for Animal Research in Uppsala.

The animals were euthanized using xylazine/ ketamine anesthesia.

For biodistribution studies, BALB/C $n u / n u$ mice were subcutaneously implanted with $10^{7}$ HER2-expressing SKOV-3 cells. Experiments were performed 2-3 wk after implantation of tumors. We evaluated the influence of coinjection of Gelofusine, the molar ratio of primary and secondary probes, the time between injection of primary and secondary probes, and the possibility of increasing the delivery of ${ }^{177} \mathrm{Lu}$ to tumors. Gelofusine is a plasma expander that reduces the renal reabsorption of many radiopeptides (25). It was found that coinjection of Gelofusine reduces renal uptake of ${ }^{177} \mathrm{Lu}-\mathrm{HP} 2$ (24).

Five mice per data point were used. The animals were injected with a predetermined amount of $\mathrm{Z}_{\text {HER2:342-SR-HP1 in the tail vein. }}$ After a preset time, each mouse was injected 
TABLE 1

Parameters Evaluated During Optimization of Pretargeting

\begin{tabular}{|c|c|c|c|c|c|}
\hline \multicolumn{2}{|c|}{$\begin{array}{l}\text { Injected mass of } \\
\text { Z }_{\text {HER2:342-SR-HP1 }}\end{array}$} & \multicolumn{2}{|c|}{$\begin{array}{l}\text { Injected mass } \\
\text { of }{ }^{177} \mathrm{Lu}-\mathrm{HP} 2 \\
\end{array}$} & \multirow{2}{*}{$\begin{array}{c}\text { Time between injection of } \\
\mathrm{Z}_{\text {HER2:342-SR-HP1 and }}{ }^{177} \text { Lu-HP2 (h) }\end{array}$} & \multirow{2}{*}{$\begin{array}{l}\text { Coinjection of Gelofusine } \\
\text { with }{ }^{177} \text { Lu-HP2 }\end{array}$} \\
\hline$\mu \mathrm{g}$ & $\mathrm{nmol}$ & $\mu g$ & $\mathrm{nmol}$ & & \\
\hline 100 & 7.6 & 1 & 0.19 & 4 & No \\
\hline 100 & 7.6 & 1 & 0.19 & 4 & Yes \\
\hline 50 & 3.8 & 1 & 0.19 & 4 & Yes \\
\hline 50 & 3.8 & 1 & 0.19 & 8 & Yes \\
\hline 50 & 3.8 & 1 & 0.19 & 16 & Yes \\
\hline None & & 1 & 0.19 & - & Yes \\
\hline 100 & 7.6 & 2 & 0.38 & 16 & Yes \\
\hline 100 & 7.6 & 3 & 0.57 & 16 & Yes \\
\hline
\end{tabular}

intravenously with $120 \mathrm{kBq}$ of ${ }^{177} \mathrm{Lu}-\mathrm{HP} 2$. The injected HP2 mass was adjusted to the predetermined amount for each group by adding unlabeled HP2 (Table 1). The animals were euthanized $1 \mathrm{~h}$ after injection of ${ }^{177} \mathrm{Lu}-\mathrm{HP} 2$, the organs were excised and weighed, and their radioactivity was measured using an automated $\gamma$-spectrometer (PerkinElmer).

\section{Biodistribution and Dosimetry}

To evaluate dosimetry, each mouse in groups of 5 was injected with $100 \mu \mathrm{g}(7.6 \mathrm{nmol})$ of $Z_{\mathrm{HER} 2: 342}-\mathrm{SR}-\mathrm{HP} 1$, followed $16 \mathrm{~h}$ later by $3 \mu \mathrm{g}$ $(120 \mathrm{kBq})$ of ${ }^{177} \mathrm{Lu}-\mathrm{HP} 2$. Biodistribution was measured at $0.3,1,4,24$, 48,144 , and $216 \mathrm{~h}$ after ${ }^{177} \mathrm{Lu}-\mathrm{HP} 2$ injection, as described above. To evaluate the dose to the renal cortex, the kidneys of 2 mice were excised $1 \mathrm{~h}$ after injection of ${ }^{177} \mathrm{Lu}-\mathrm{HP} 2(3 \mu \mathrm{g}, 1 \mathrm{MBq})$ and $20-\mu \mathrm{m}$ cryosections were prepared. The distribution of radioactivity in the sections was measured by digital autoradiography using a Cyclone Storage Phosphor System (PerkinElmer) (Supplemental Fig. 1; supplemental materials are available at http://jnm.snmjournals.org). Specific $\mathrm{S}$ factors for radioactivity uptake in the renal cortex were determined with a small-scale radiation transport calculation in Monte Carlo N-Particle code, version 6 . The cortex was modeled as a 1-mmthick rim inside a $4.8 \times 5.7 \times 9.8 \mathrm{~mm}$ ellipsoid. Absorbed doses were calculated using the RADAR realistic mouse phantom (26). Absorbed doses to tumors were calculated with the spheric node $\mathrm{S}$ values (27) without considering the contribution to the tumor-absorbed dose from activity in the mouse. The absorbed dose to spleen can well be considered representative for calculating the absorbed dose from activity in the mouse to a tumor in the abdomen. When we perform these calculations, the absorbed cross-dose to the spleen from other organs is on the order of $8.5 \mathrm{mGy}$, which would increase the absorbed dose to tumors by $0.05 \%$. Considering all uncertainties in the absorbed dose calculations, this percentage can be considered completely negligible. Tumor volumes were taken from data on SKOV-3 xenograft growth in untreated control mice from previous studies (Supplemental Fig. 2).

\section{Experimental Therapy}

To evaluate pretargeted therapy, $10^{7}$ SKOV-3 cells per mouse were implanted subcutaneously in the abdomen. At least twice a week, the mice were weighed and visually inspected, and tumors were measured using electronic calipers. Tumor volumes $\left(\mathrm{mm}^{3}\right)$ were calculated as [length $(\mathrm{mm})] \times[\text { width }(\mathrm{mm})]^{2} \times 0.5$. The animals were euthanized when tumors reached a size of $1,000 \mathrm{~mm}^{3}$ or became ulcerated, or if an animal's weight dropped by more than $10 \%$ during $1 \mathrm{wk}$ or by more than $15 \%$ since the study began. After euthanasia, tumors and kidneys were excised for subsequent histologic evaluation. 
Treatment started $14 \mathrm{~d}$ after tumor implantation, when the average tumor volume was $83 \pm 43 \mathrm{~mm}^{3}$. The mice were randomly divided into 4 groups of 10 animals each. Those in the treatment group were injected with $100 \mu \mathrm{g}(7.6 \mathrm{nmol})$ of $\mathrm{Z}_{\mathrm{HER} 2: 342}$-SR-HP1, followed $16 \mathrm{~h}$ later by $3.5 \mu \mathrm{g}(0.65 \mathrm{nmol}) / 16 \mathrm{MBq}$ of ${ }^{177} \mathrm{Lu}-\mathrm{HP} 2$ in a $100-\mu \mathrm{L}$ solution containing $2 \%$ bovine serum albumin and $4 \mathrm{mg}$ of Gelofusine. Control group 1 received vehicle alone. To evaluate the influence of nonlabeled $\mathrm{Z}_{\mathrm{HER} 2: 342^{-}} \mathrm{SR}-\mathrm{HP} 1$ on tumor growth, a control group 2 was injected with $100 \mu \mathrm{g}(7.6 \mathrm{nmol})$ of $\mathrm{Z}_{\mathrm{HER} 2: 342}$-SR-HP1 only. To evaluate the effect of nontargeted radiolabeled secondary agent, a control group 3 was injected with ${ }^{177} \mathrm{Lu}-\mathrm{HP} 2(3.5 \mu \mathrm{g} / 16 \mathrm{MBq})$ only. In total, this procedure was repeated 6 times at $7-d$ intervals. The total injected radioactivity was $96 \mathrm{MBq}$ per mouse.

To confirm tumor targeting, SPECT/CT imaging was performed. Five mice were imaged $30 \mathrm{~h}$ after injection of $16 \mathrm{MBq}$ of ${ }^{177} \mathrm{Lu}-\mathrm{HP} 2$. Imaging was performed using a nanoScan SC device (Mediso Medical Imaging Systems) under sevoflurane anesthesia. CT acquisitions were performed using a CT energy peak of $50 \mathrm{keV}, 670 \mu \mathrm{A}, 480$ projections, and a 2.3-min acquisition time. SPECT helical scans were acquired with the following parameters: a ${ }^{177} \mathrm{Lu}$ energy window (50.49-61.71, 101.61-124.19, and 187.56-229.24 keV), 110 projections, a $256 \times$ 256 matrix, and a 20-min acquisition time. CT raw files were reconstructed using Nucline software (version 2.03; Mediso Medical Imaging Systems). SPECT raw data were reconstructed using Tera-Tomo (Mediso Medical Imaging Systems) 3-dimensional SPECT.

\section{Histologic and Immunohistochemical Evaluation}

The xenografts and kidneys from each mouse in the therapy experiment were formalin-fixed and paraffin-embedded using standard procedures. For histologic evaluation, $4-\mu \mathrm{m}$ sections were stained with hematoxylin and eosin. To evaluate HER2 expression after therapy, an immunohistochemical evaluation was performed using HercepTest Assay (Dako).

\section{Statistical Analysis}

Prism software (GraphPad Software, Inc.) was used for statistical analysis. Biodistribution data were analyzed using a 2-tailed unpaired $t$ test to find significant differences $(P<0.05)$ when 2 groups were compared. When 3 or more groups were compared, a 2-way ANOVA with Bonferroni testing for multiple comparisons was applied. Survival data were analyzed using Prism software.

\section{RESULTS}

\section{Factors Influencing Pretargeting}

The results of the experiments elucidating factors influencing pretargeting are presented in Fig. 2. The dosing and timing used in pretargeting of ${ }^{111} \mathrm{In}-\mathrm{HP} 2$ (Fig. 2A) (23) resulted in higher renal uptake than tumor uptake. Coinjection of Gelofusine with ${ }^{177} \mathrm{Lu}-$ HP2 did not decrease renal uptake. A doubling of the ${ }^{177} \mathrm{Lu}-\mathrm{HP} 2-$

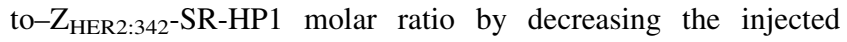
$\mathrm{Z}_{\mathrm{HER} 2: 342^{-S R}-\mathrm{HP} 1 \text { mass significantly reduced }(P<0.05) \text { retention }}$ of activity in blood and uptake in lung, liver, and, most importantly, kidneys (Fig. 2B), whereas tumor uptake of ${ }^{177} \mathrm{Lu}-\mathrm{HP} 2$ tended to increase. Increasing the interval between injection of

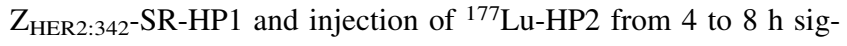
nificantly reduced $(P<0.05)$ retention of activity in blood and uptake in lung, liver, spleen, and kidneys (Fig. 2C). Renal uptake

TABLE 2

Biodistribution and Dosimetry of ${ }^{177} \mathrm{Lu}-\mathrm{HP} 2$ Injected 16 Hours After $Z_{\text {HER2:342-SR-HP1 in BALB/C nu/nu Mice Bearing }}$ SKOV-3 Xenografts

\begin{tabular}{|c|c|c|c|c|c|c|c|c|}
\hline \multirow[b]{2}{*}{ Site } & \multicolumn{7}{|c|}{ Uptake } & \multirow{2}{*}{$\begin{array}{c}\text { Estimated mean } \\
\text { absorbed dose } \\
\text { (mGy/MBq) }\end{array}$} \\
\hline & $0.3 \mathrm{~h}$ & $1 \mathrm{~h}$ & $4 \mathrm{~h}$ & $24 \mathrm{~h}$ & $48 \mathrm{~h}$ & $144 \mathrm{~h}$ & $216 \mathrm{~h}$ & \\
\hline Blood & $2.2 \pm 0.3$ & $0.28 \pm 0.07$ & $0.019 \pm 0.003$ & $0.010 \pm 0.005$ & $0.008 \pm 0.001$ & & & 4.0 \\
\hline Heart & $0.8 \pm 0.1$ & $0.12 \pm 0.06$ & $0.03 \pm 0.01$ & $0.016 \pm 0.007$ & $0.02 \pm 0.02$ & & & 5.4 \\
\hline Lung & $1.9 \pm 0.2$ & $0.41 \pm 0.07$ & $0.15 \pm 0.01$ & $0.06 \pm 0.01$ & $0.05 \pm 0.01$ & & & 14.4 \\
\hline Salivary gland & $0.9 \pm 0.2$ & $0.2 \pm 0.1$ & $0.06 \pm 0.02$ & $0.04 \pm 0.01$ & $0.03 \pm 0.01$ & & & 9.3 \\
\hline Liver & $0.56 \pm 0.06$ & $0.20 \pm 0.02$ & $0.15 \pm 0.04$ & $0.12 \pm 0.03$ & $0.11 \pm 0.05$ & $0.06 \pm 0.03$ & $0.05 \pm 0.02$ & 13.3 \\
\hline Spleen & $0.59 \pm 0.08$ & $0.14 \pm 0.03$ & $0.07 \pm 0.02$ & $0.08 \pm 0.02$ & $0.06 \pm 0.01$ & & & 12.8 \\
\hline Pancreas & $0.5 \pm 0.2$ & $0.10 \pm 0.02$ & $0.024 \pm 0.007$ & $0.017 \pm 0.008$ & $0.02 \pm 0.01$ & & & 4.0 \\
\hline Stomach & $1.1 \pm 0.2$ & $0.19 \pm 0.03$ & $0.10 \pm 0.03$ & $0.05 \pm 0.03$ & $0.033 \pm 0.006$ & & & 12.3 \\
\hline Small intestine & $0.7 \pm 0.1$ & $0.14 \pm 0.02$ & $0.05 \pm 0.02$ & $0.03 \pm 0.02$ & $0.027 \pm 0.007$ & & & 7.0 \\
\hline Large intestine & $0.9 \pm 0.3$ & $0.18 \pm 0.03$ & $0.07 \pm 0.03$ & $0.03 \pm 0.02$ & $0.05 \pm 0.02$ & & & 10.4 \\
\hline Kidney & $12 \pm 2$ & $5 \pm 1$ & $4 \pm 1$ & $3 \pm 1$ & $2.3 \pm 0.7$ & $0.7 \pm 0.2$ & $0.5 \pm 0.2$ & 205.6 \\
\hline Kidney cortex ${ }^{*}$ & & & & & & & & 267 \\
\hline Tumor & $15 \pm 3$ & $20 \pm 5$ & $17 \pm 3$ & $15 \pm 2$ & $12 \pm 1$ & $3.4 \pm 0.6$ & $1.8 \pm 0.3$ & $1,075.4$ \\
\hline Skin & $1.8 \pm 0.3$ & $0.3 \pm 0.1$ & $0.13 \pm 0.03$ & $0.10 \pm 0.05$ & $0.08 \pm 0.03$ & $0.03 \pm 0.01$ & $0.04 \pm 0.02$ & \\
\hline Muscle & $0.6 \pm 0.1$ & $0.10 \pm 0.04$ & $0.03 \pm 0.01$ & $0.014 \pm 0.003$ & $0.019 \pm 0.008$ & & & \\
\hline Bone & $0.64 \pm 0.07$ & $0.13 \pm 0.04$ & $0.04 \pm 0.01$ & $0.03 \pm 0.01$ & $0.03 \pm 0.01$ & & & 6.8 \\
\hline Brain & $0.07 \pm 0.01$ & $0.01 \pm 0.00$ & $0.004 \pm 0.002$ & $0.002 \pm 0.002$ & $0.002 \pm 0.001$ & & & 0.6 \\
\hline
\end{tabular}

${ }^{*}$ Estimation based on autoradiography data.

Uptake is average percentage injected activity per gram $\pm \operatorname{SD}(n=5)$. 


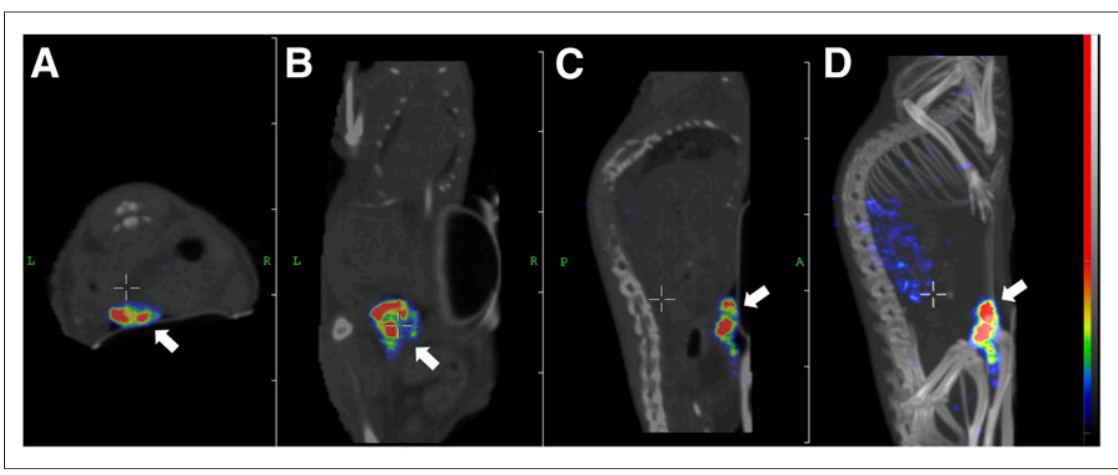

FIGURE 3. Transversal (A), coronal (B), and sagittal (C) SPECT/CT images and maximum-

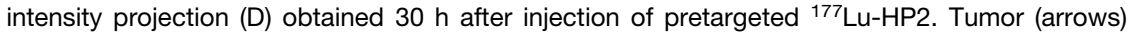
was located on abdomen.

was significantly lower $(P<0.05)$ after $16 \mathrm{~h}$ than after $8 \mathrm{~h}$. There was no significant difference between tumor uptake when the interval from the $\mathrm{Z}_{\mathrm{HER} 2: 342}$-SR-HP1 injection to the ${ }^{177} \mathrm{Lu}-\mathrm{HP} 2$ injection was 4, 8, or $16 \mathrm{~h}$ (Fig. 2C). Upscaling experiments (Fig. 2D) showed no significant changes in the biodistribution of ${ }^{177} \mathrm{Lu}-$ HP2 when the injected mass of both $\mathrm{Z}_{\mathrm{HER} 2: 342}$-SR-HP1 and ${ }^{177} \mathrm{Lu}-$ HP2 was doubled. Further, a 1.5-fold increase in the ${ }^{177} \mathrm{Lu}-\mathrm{HP} 2$ mass did not significantly change tumor or renal uptake but enabled an increase in the radioactivity delivered to tumors.

Tumor uptake of ${ }^{177} \mathrm{Lu}-\mathrm{HP} 2$ with no preinjection of $\mathrm{Z}_{\mathrm{HER} 2: 342^{-}}$ SR-HP1 was $0.2 \% \pm 0.1 \%$ of the injected activity per gram, which is significantly lower $\left(P<5 \times 10^{-5}\right)$ than after injection of any tested mass of $Z_{\mathrm{HER} 2: 342^{-}} \mathrm{SR}-\mathrm{HP} 1$ at any tested time point. This finding suggests that tumor uptake of ${ }^{177} \mathrm{Lu}-\mathrm{HP} 2$ depends on Affibody-directed PNA-mediated pretargeting.

\section{Biodistribution and Dosimetry}

Biodistribution and dosimetry data are presented in Table 2 . The data demonstrate rapid clearance of ${ }^{177} \mathrm{Lu}-\mathrm{HP} 2$ from most tissues; for example, clearance from blood had a 13-min (range, 10$17 \mathrm{~min}$ ) half-life. The only tissues with prominent uptake were kidneys and tumors. However, tumor uptake was already 4-fold higher than renal uptake at $1 \mathrm{~h}$ after injection, as $84 \%$ of renal uptake cleared with a 15-min half-life whereas the tumor clearance half-life was $63 \mathrm{~h}$. The time-integrated activity coefficient per gram of tissue was higher for tumors than for kidneys, blood, and bone, at 5.2-, 254- and 147-fold, respectively. Accordingly, the calculated mean absorbed dose to tumors was appreciably higher than that to any normal organ or tissue (Table 2).

\section{Experimental Therapy}

Imaging performed during experimental therapy (Fig. 3) demonstrated a high accumulation of ${ }^{177} \mathrm{Lu}$ in tumors. The radioactivity concentration in kidneys was about 5-fold lower than in tumors, and the concentrations in other tissues were much lower.

Survival data are presented in Figure 4A. Pretargeted therapy clearly improved survival in mice bearing HER2-expressing xenografts. Median survival in the control groups was 32,37 , and $37 \mathrm{~d}$ for treatment with ${ }^{177} \mathrm{Lu}-\mathrm{HP} 2$ alone, $\mathrm{Z}_{\mathrm{HER} 2: 342-\mathrm{SR}-\mathrm{HP} 1}$ alone, and phosphate-buffered saline, respectively. There was no significant difference in median survival among these groups. Median survival in the group receiving $16 \mathrm{MBq} / 3.5 \mu \mathrm{g}$ of ${ }^{177} \mathrm{Lu}-\mathrm{HP} 216 \mathrm{~h}$ after $100 \mu \mathrm{g}(7.6 \mathrm{nmol})$ of $\mathrm{Z}_{\mathrm{HER} 2: 342^{-}} \mathrm{SR}-\mathrm{HP} 1$ was $66 \mathrm{~d}$, which was significantly longer $(P<0.005)$ than in any of the control groups. In this group, an animal with a $157-\mathrm{mm}^{3}$ tumor at the start of therapy showed complete remission at day 61, which lasted until the end of the study (day 95). A detailed investigation of the body of this mouse did not reveal any visceral residual tumor or metastases. The absorbed dose to tumor was $102 \mathrm{~Gy}$.

The pretargeted therapy was well tolerated. The appearance of the skin, fat pads, and eyes did not differ between treated and untreated mice, and there was no behavior indicating pain or suffering. The average animal weight was comparable to that of mice injected with phosphate-buffered saline (Fig. 4B).

\section{Histologic and Immunohistochemical Evaluation}

Evaluation of kidneys did not indicate any toxic impact by the treatment. The renal parenchymal structures, including glomeruli, tubuli, and vessels, were well preserved (Fig. 5). Histologic analysis of the xenografts showed poorly differentiated cancer with a solid morphology and variable areas of necrosis. Comparison of all 4 treatment groups did not reveal any difference in histologic growth pattern or degree of necrosis (Fig. 5). Immunohistochemical analysis of tumors demonstrated moderate to strong membrane staining in almost all viable tumor cells (Fig. 5). The staining pattern revealed no difference in HER2 expression among the 4 treatment arms.

\section{DISCUSSION}

Pretargeting strategies were initially created to overcome limitations associated with the bulkiness of immunoglobulin-based targeting vectors $(1)$. We $(23,28)$ and others $(29)$ proposed the use of pretargeting to compensate for undesirable high renal reabsorption of small targeting proteins. This study demonstrated that pretargeting can be efficient in reducing renal uptake: under optimized conditions, renal uptake of ${ }^{177} \mathrm{Lu}-\mathrm{HP} 2$ was $4 \% \pm$ $1 \%$ of the injected activity per gram at $4 \mathrm{~h}$ after injection. This uptake is 70-fold lower than that of ${ }^{177} \mathrm{Lu}$-labeled parental Affibody molecules (30).

However, our study highlights some pitfalls in developing pretargeted therapies. For example, renal uptake was higher than
FIGURE 4. Survival of BALB/C nu/nu mice treated with $Z_{\text {HER2:342-SR-HP1 (ZHP1) plus }{ }^{177} \text { Lu }}$

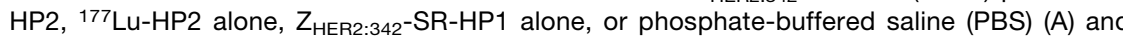
average animal weight during therapy (B).

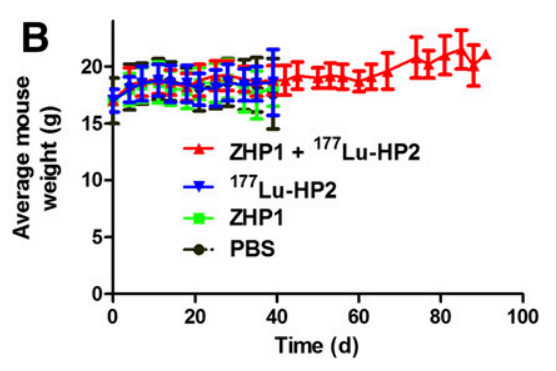




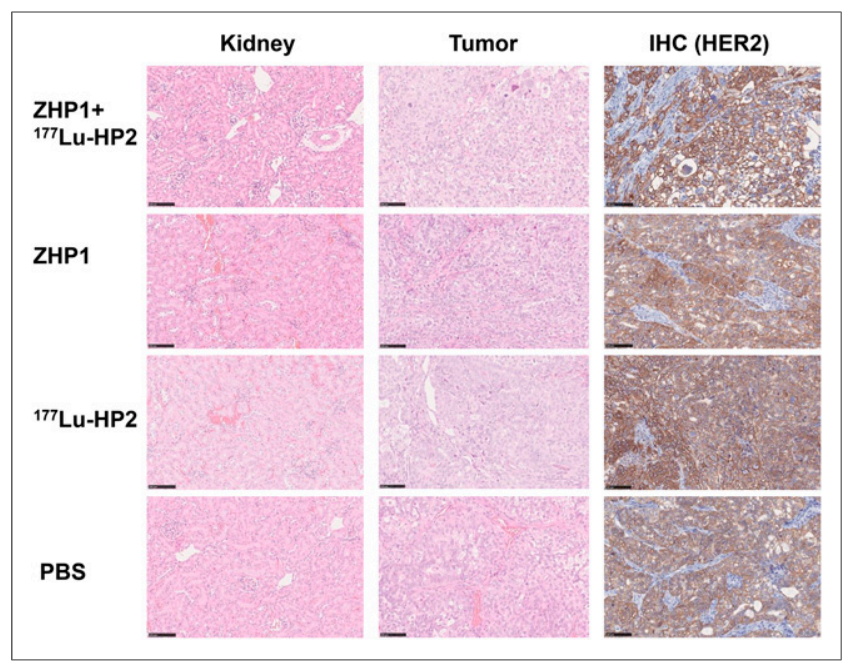

FIGURE 5. Representative images of hematoxylin- and eosin-stained renal cortex and tumor, and immunohistochemical analysis (IHC) of HER2 expression, in tumors of mice treated using pretargeting. IHC = immunohistochemistry; PBS = phosphate-buffered saline; ZHP1 = $\mathrm{Z}_{\text {HER2:342-SR-HP1. }}$.

tumor uptake (Fig. 2A) when we applied the same dosing and timing using ${ }^{177} \mathrm{Lu}-\mathrm{HP} 2$, which provided higher uptake of ${ }^{111} \mathrm{In}-$ HP2 in tumors than in kidneys (23). This finding was surprising since the data for ${ }^{177} \mathrm{Lu}-\mathrm{HP} 2$ alone (24) demonstrated that renal uptake of ${ }^{177} \mathrm{Lu}-\mathrm{HP} 2$ was not higher than that of ${ }^{111} \mathrm{In}-\mathrm{HP} 2$. Furthermore, Gelofusine was not efficient for reducing renal uptake in pretargeting settings (Fig. 2A) but was efficient for ${ }^{177} \mathrm{Lu}-\mathrm{HP} 2$ alone (24). On the other hand, Gelofusine does not reduce renal uptake of Affibody molecules (17). Thus, ${ }^{177} \mathrm{Lu}-\mathrm{HP} 2$ was most likely taken up by kidneys as an adduct with $\mathrm{Z}_{\mathrm{HER} 2: 342}$-SR-HP1 formed by interaction with residual primary agent in blood. The reduction in renal uptake after an increase in the ${ }^{177} \mathrm{Lu}-\mathrm{HP} 2 /$ $\mathrm{Z}_{\mathrm{HER} 2: 342}$-SR-HP1 molar ratio confirmed this possibility. The most efficient way to reduce renal uptake was to increase the time between injection of the primary and secondary probes, permitting

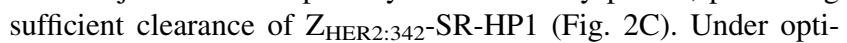
mized conditions, the PNA-mediated pretargeting provided more than a 5-fold higher absorbed dose to tumors than to kidneys. Earlier, we evaluated another approach to Affibody-based pretargeted therapy, the use of a bioorthogonal reaction between transcyclooctene and tetrazine (28). In that case, the tumor-absorbed dose exceeded the kidney-absorbed dose by approximately 1.4fold under optimized conditions. Apparently, the use of PNAPNA interaction provides much more favorable dosimetry for Affibody-based pretargeting and is preferable.

The efficacy of pretargeted therapy was shown in this study by a significant extension of survival. No pathologic changes in kidneys were observed (Fig. 5). This finding agrees with the current practice of clinical radionuclide therapy, which is given with palliative intent. An increase in absorbed dose rate should increase the efficacy of tumor treatment, as might be achieved by the use of $\alpha$-emitting ${ }^{225} \mathrm{Ac}$ or no-carrier-added ${ }^{177} \mathrm{Lu}$.

Our approach can easily be applied to other ESPs. The use of an optimized protocol (24) should provide efficient ligation of hybridization probes to any ESP containing an LPXTG tag, potentially enabling development of pretargeted radionuclide therapy based on ESPs having high renal reabsorption, such as fibronectin domains. In all cases, site-specific coupling of HP1 would provide well-defined homogeneous constructs with reproducible biodistribution.

\section{CONCLUSION}

Optimization of the timing and dose of injection of Affibody molecules containing a PNA-based hybridization probe and a ${ }^{177} \mathrm{Lu}$-labeled complementary probe enabled delivery of a tumorabsorbed dose that exceeded the dose absorbed by kidneys. Affibody-based PNA-mediated pretargeted radionuclide therapy significantly increased survival in mice bearing human HER2-expressing cancer xenografts.

\section{DISCLOSURE}

This research was financially supported by grants from the Swedish Cancer Society, the Swedish Research Council, the ProNova VINN Excellence Centre for Protein Technology, and the Swedish Agency for Innovation, Vinnova. No other potential conflict of interest relevant to this article was reported.

\section{REFERENCES}

1. Stoldt HS, Aftab F, Chinol M, et al. Pretargeting strategies for radio-immunoguided tumour localisation and therapy. Eur J Cancer. 1997;33:186-192.

2. Pouget JP, Navarro-Teulon I, Bardiès M, et al. A clinical radioimmunotherapy: the role of radiobiology. Nat Rev Clin Oncol. 2011;8:720-734.

3. van de Watering FC, Rijpkema M, Robillard M, Oyen WJ, Boerman OC. Pretargeted imaging and radioimmunotherapy of cancer using antibodies and bioorthogonal chemistry. Front Med (Lausanne). 2014;1:44.

4. Altai M, Membreno R, Cook B, Tolmachev V, Zeglis BM. A primer on pretargeted imaging and therapy. J Nucl Med. 2017;58:1553-1559.

5. Vazquez-Lombardi R, Phan TG, Zimmermann C, Lowe D, Jermutus L, Christ D. Challenges and opportunities for non-antibody scaffold drugs. Drug Discov Today. 2015;20:1271-1283.

6. Stern LA, Case BA, Hackel BJ. Alternative non-antibody protein scaffolds for molecular imaging of cancer. Curr Opin Chem Eng. 2013;2:425-432.

7. Feldwisch J, Tolmachev V. Engineering of affibody molecules for therapy and diagnostics. Methods Mol Biol. 2012;899:103-126.

8. Hackel BJ, Kimura RH, Gambhir SS. Use of ${ }^{64} \mathrm{Cu}$-labeled fibronectin domain with EGFR-overexpressing tumor xenograft: molecular imaging. Radiology. 2012;263:179-188.

9. Goldstein R, Sosabowski J, Livanos M, et al. Development of the designed ankyrin repeat protein (DARPin) G3 for HER2 molecular imaging. Eur J Nucl Med Mol Imaging. 2015;42:288-301.

10. Garousi J, Lindbo S, Nilvebrant J, et al. ADAPT, a novel scaffold protein-based probe for radionuclide imaging of molecular targets that are expressed in disseminated cancers. Cancer Res. 2015;75:4364-4371.

11. Nord K, Gunneriusson E, Ringdahl J, Ståhl S, Uhlén M, Nygren PA. Binding proteins selected from combinatorial libraries of an alpha-helical bacterial receptor domain. Nat Biotechnol. 1997;15:772-777.

12. Ståhl S, Gräslund T, Eriksson Karlström A, Frejd FY, Nygren PÅ, Löfblom J. Affibody molecules in biotechnological and medical applications. Trends Biotechnol. 2017;35:691-712.

13. Bartley AN, Washington MK, Colasacco C, et al. HER 2 testing and clinical decision making in gastroesophageal adenocarcinoma: guideline from the college of American Pathologists, American Society for Clinical Pathology, and the American Society of Clinical Oncology. J Clin Oncol. 2017;35: $446-464$.

14. Wolff AC, Hammond ME, Hicks DG, et al. Recommendations for human epidermal growth factor receptor 2 testing in breast cancer: American Society of Clinical Oncology/College of American Pathologists clinical practice guideline update. J Clin Oncol. 2013;31:3997-4013.

15. Sörensen J, Sandberg D, Sandström M, et al. First-in-human molecular imaging of HER2 expression in breast cancer metastases using the ${ }^{111}$ In-ABY-025 affibody molecule. J Nucl Med. 2014;55:730-735.

16. Sörensen J, Velikyan I, Sandberg D, et al. Measuring HER2-receptor expression in metastatic breast cancer using $\left[{ }^{68} \mathrm{Ga}\right] \mathrm{ABY}-025$ affibody PET/CT. Theranostics. 2016;6:262-271. 
17. Altai M, Varasteh Z, Andersson K, Eek A, Boerman O, Orlova A. In vivo and in vitro studies on renal uptake of radiolabeled affibody molecules for imaging of HER2 expression in tumors. Cancer Biother Radiopharm. 2013;28:187-195.

18. Egholm M, Buchardt O, Christensen L, et al. PNA hybridizes to complementary oligonucleotides obeying the Watson-Crick hydrogen-bonding rules. Nature. 1993;365:566-568.

19. Cutrona G, Boffa LC, Mariani MR, et al. The peptide nucleic acid targeted to a regulatory sequence of the translocated c-myc oncogene in Burkitt's lymphoma lacks immunogenicity: follow-up characterization of PNAEmu-NLS. Oligonucleotides. 2007;17:146-150.

20. Boffa LC, Menichini P, Bolognesi C, et al. Lack of mutagenicity and clastogenicity of Emu-NLS targeted to a regulatory sequence of the translocated c-myc oncogene in Burkitt's lymphoma. Mutat Res. 2007;628:129-137.

21. Rusckowski M, Qu T, Chang F, Hnatowich DJ. Pretargeting using peptide nucleic acid. Cancer. 1997;80:2699-2705.

22. Westerlund K, Honarvar H, Tolmachev V, Eriksson Karlström A. Design, preparation, and characterization of PNA-based hybridization probes for affibodymolecule-mediated pretargeting. Bioconjug Chem. 2015;26:1724-1736.

23. Honarvar H, Westerlund K, Altai M, et al. Feasibility of affibody molecule-based PNA-mediated radionuclide pretargeting of malignant tumors. Theranostics. 2016;6: 93-103.
24. Altai M, Westerlund K, Velletta J, Mitran B, Honarvar H, Eriksson Karlström A. Evaluation of affibody molecule-based PNA-mediated radionuclide pretargeting: development of an optimized conjugation protocol and ${ }^{177} \mathrm{Lu}$ labeling. Nucl Med Biol. 2017;54:1-9.

25. Rolleman EJ, Melis M, Valkema R, Boerman OC, Krenning EP, de Jong M. Kidney protection during peptide receptor radionuclide therapy with somatostatin analogues. Eur J Nucl Med Mol Imaging. 2010;37:1018-1031.

26. Keenan MA, Stabin MG, Segars WP, Fernald MJ. RADAR realistic animal model series for dose assessment. J Nucl Med. 2010;51:471-476.

27. Stabin MG, Konijnenberg MW. Re-evaluation of absorbed fractions for photons and electrons in spheres of various sizes. J Nucl Med. 2000;41:149-160.

28. Altai M, Perols A, Tsourma M, et al. Feasibility of affibody-based bioorthogonal chemistry-mediated radionuclide pretargeting. J Nucl Med. 2016;57: 431-436.

29. van Duijnhoven SM, Rossin R, van den Bosch SM, Wheatcroft MP, Hudson PJ, Robillard MS. Diabody pretargeting with click chemistry in vivo. J Nucl Med. 2015;56:1422-1428.

30. Fortin MA, Orlova A, Malmström PU, Tolmachev V. Labelling chemistry and characterization of $\left[{ }^{90 Y / 177} \mathrm{Lu}\right]-D O T A-Z H E R 2: 342-3$ Affibody molecule, a candidate agent for locoregional treatment of urinary bladder carcinoma. Int $\mathrm{J} \mathrm{Mol}$ Med. 2007;19:285-291. 